\title{
Repeated application of autologous bone marrow-derived stem cell therapy in patients with severe Buerger's disease
}

\author{
Zoltan Boda $^{1}$, K. Razso ${ }^{1}$, M. Szarvas ${ }^{1}$, Zs. Olah ${ }^{1}$, P. Ilonczai ${ }^{1}$, Z. Vereb ${ }^{2}$, E. Rajnavolgyi ${ }^{2}$ \\ ${ }^{1}$ Department of Internal Medicine, University of Debrecen, Debrecen, Hungary; zboda@med.unideb.hu \\ ${ }^{2}$ Department of Immunology, University of Debrecen, Debrecen, Hungary
}

Received February 18 2011; revised March 21 2011; accepted March 282011.

\begin{abstract}
Stem cell therapy (SCT) is a promising and prospective approach in the treatment of patients with severe peripheral arterial disorders, primarily with Buerger's disease. However, very little is known about the duration of the effect of SCT, and to our best knowledge no data are available on the efficacy and safety of repeated SCT in patients with Buerger's disease. Here we report on two patients with severe Buerger's disease, who received repeated autologous bone marrow-derived stem cell therapy. Our results show that a second SCT, applied to a previously treated leg $\mathbf{3 0}$ or $\mathbf{3 6}$ months after the first treatment was efficient in both cases. After twelve months, the clinical state of the repeatedly treated lower limb improved spectacularly and non-healing ulcers healed more rapidly than after the first SCT. No severe adverse events were detected. Thus repeated SCT offers a safe and efficient treatment option for relapsing patients at the advanced stage of Buerger's disease.
\end{abstract}

Keywords: Buerger's Disease; Non Healing Ulcer; Repeated Bone Marrow-Derived Stem Cell Therapy

\section{INTRODUCTION}

Recent publications have proved the efficacy and safety of stem cell therapy (SCT) in patients with severe

Presented at the 54th Annual Meeting of German Society of Thrombosis Haemostasis (GTH), Nuremberg, February 2010.

Fundings: The present study was supported by the EEA/Norway Grants HU-0046/NA/2006-2/ÖP-9 entitled Development of novel stem cell-based therapies for the treatment of advanced peripheral arterial disease and by the TAMOP 4.2.2-08/1-2008-0015 EU supported NFÜ program entitled Establishing a stem cell and gene therapy. Research Centre at the University of Debrecen.
Buerger's disease [1-14]. However, very little information is available on the duration of the beneficial effect of SCT [12], and according to our best knowledge no data exist on the efficacy and safety of repeated SCT carried out in patients with relapsing or worsening clinical states in severe Buerger's disease. Here we report on two cases, where the lower limb of patients with Buerger's disease, previously treated by autologous bone marrow-derived stem cell therapy (ABMSCT) [9], have been re-treated with freshly isolated autologous bone marrow derived isolated $\mathrm{CD}_{34}{ }^{+}$cells. Both patients relapsed at 30 - 36 months after the first ABMSCT. As they responded to the first treatment, we decided to repeat ABMSCT with freshly isolated bone marrow derived $\mathrm{CD}_{34}{ }^{+}$cells by using the same method as described in our previous publication [9].

\section{MATERIALS AND METHODS}

As it is a repeated stem cell therapy we refer to our previous publication [9].

\section{RESULTS}

\subsection{Case Reports}

Patient 1 was a 59-year-old man with Buerger's disease since 1976. He had rest pain, $50 \mathrm{~m}$ walking distance without pain, and 2 large $\left(8 \mathrm{~cm}^{2}\right)$ and deep non-healing ulcers on both feet. He had sympathectomy on both sides (1982, 1983). Amputation of the right toe II and left toe I was carried out in 1991, and the amputation of the left toe III was done in 1995. Disease stage was Lerishe/Fontain-IV before ABMSCT. As his left lower limb was in a worse condition, we treated it by ABMSCT in August 2006. The clinical state of this limb is still excellent (rest pain disappeared, the ischaemic ulcer healed and has not relapsed so far), whereas the clinical state of the untreated right limb became worse (rest pain in- 
creased, the ischaemic ulcer got larger and deeper). In April 2007 ABMSCT was carried out on his right foot (Figure 1 (a)) and nine months later we observed significant improvement of the treated right foot (Figure 1 (b)). In October 2009, 30 months after the second SCT the condition of his right limb relapsed with a large and deep ischaemic ulcer at the same place (Figure 1 (c)). His good response to previous treatments prompted us to repeat ABMSCT on his right foot. Twelve months after the repeated treatment, a significant improvement was observed both in the general angiological state of the limb and in the size of the ischaemic ulcer (Figure 1 (d)).

Patient 2 was a 40-year-old man with Buerger's disease since 1991 . He had rest pain, < $50 \mathrm{~m}$ walking distance without pain, and a large and deep non-healing ulcer $\left(36 \mathrm{~cm}^{2}\right)$ on his left foot. He had sympatectomy on both sides (1994 and 1995). He underwent amputations of his left toe I-III and right toe I-II (1994), and left finger III (1999). Disease stage was Lerishe/Fontain-IV before ABMSCT. SCT was given in September 2006

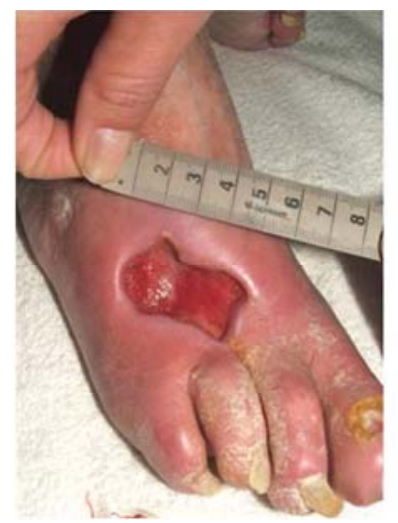

(a)

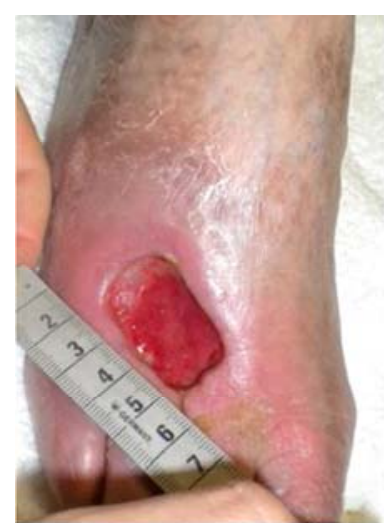

(c)

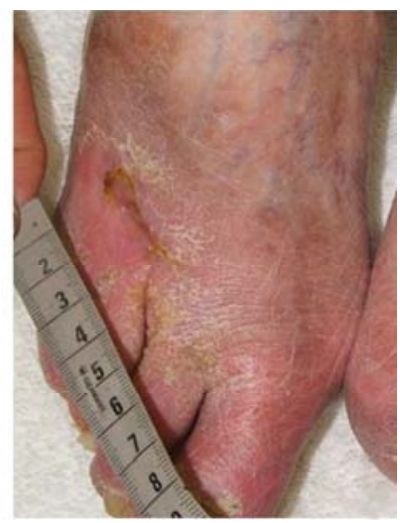

(b)

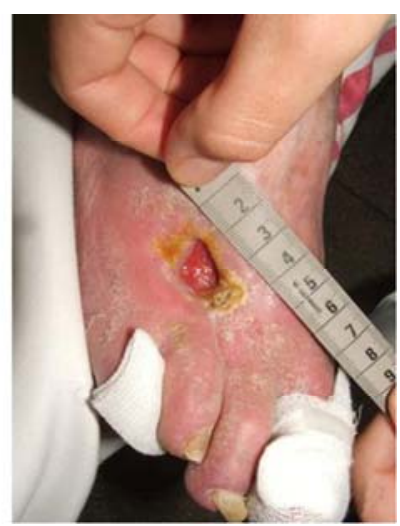

(d)
(Figure 2 (a)) and after 12 months the clinical state of the treated limb improved significantly as the non-healing ulcer healed and rest pain disappeared (Figure 2 (b)). In October 2009, 36 months after SCT a large and deep ischaemic ulcer re-appeared just at the same place as before (Figure 2 (c)). Taken his previous clinical response to therapy, we repeated ABMSCT and six months after the treatment we detected complete healing of the ischaemic ulcer (Figure 2 (d)). Simultaneously the symptoms in the limb improved significantly.

\section{DISCUSSION}

Amputation is the only option for relief of rest pain or large and deep ischaemic ulcers in patients with severe Buerger's disease. No effective blood-flow enhancement therapies are available for these patients. ABMSCT is a promising and prospective approach in the treatment of severe peripheral arterial disorders, in particular in Buerger's disease. All of the published clinical trials have reported on the affectivity and safety of ABMSCT

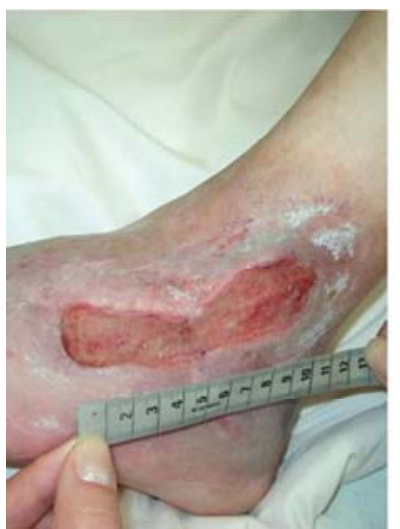

(a)

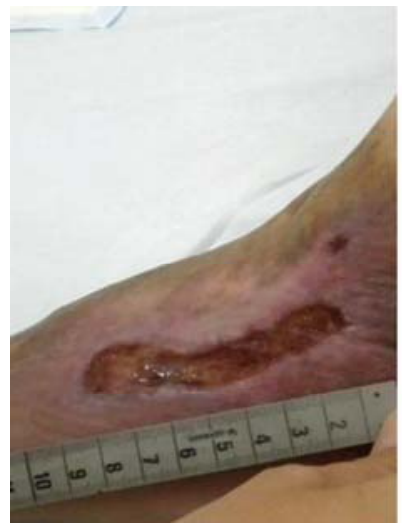

(c)

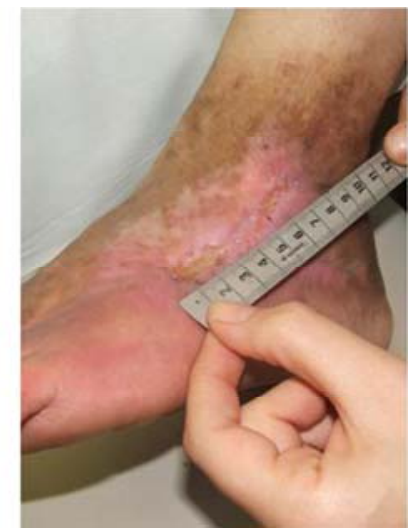

(b)

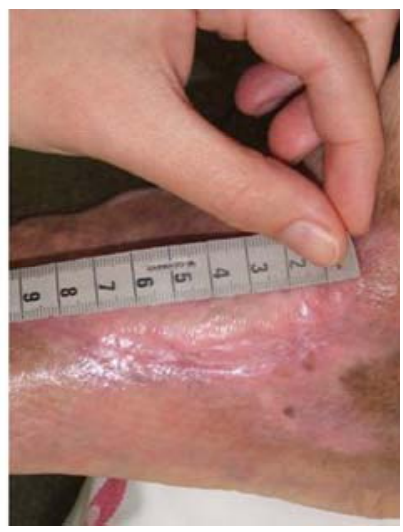

(d)
Figure 1. Repeated ABMSCT in a patient (D. A. 59 ys) with severe Buerger's disease. (a) Before first ABMSCT, (b) 9 months after first ABMSCT, (c) Relapse, 30 months after first ABMSCT, D: 6 months after second ABMSCT.
Figure 2. Repeated ABMSCT in a patient (F.T. 40 ys) with severe Buerger's disease. (a) before first ABMSCT, (b) after 12 months of first ABMSCT, (c) relapse, 36 months after first ABMSCT, (d) 6 months after second ABMSCT. 
in Buerger's disease even though the variation of the cell populations and protocols used for the therapy [1-16].

It is well established that the bone marrow contains multiple stem- and progenitor cells with the potential to support vessel regeneration. As we proposed previously [9], when these cells interact with tissue- and immune cells in the inflammed milieu of the obstructed vessel, they very likely acquire novel activities and trigger a cascade of local events that support vasculogenesis and wound healing. These complex mechanisms may involve controlled production of soluble factors, regulation of endothelial cell functions, formation of new vessels, tissue regeneration, and inhibition of inflammation $[17,18]$. These events altogether may support increased blood flow, healing of ischaemic ulcers and ultimately protect the leg from amputation.

Recently, Fadini et al. collected clinical data of 700 patients with peripheral arterial disorders treated by SCT [15]. In patients with thromboangiitis obliterans (TAO) showed larger benefits from SCT than patients with atherosclerosis obliterans (ASO). The survival rate and amputation-free state were markedly better in patients with TAO than those in patients with ASO (100\% vs. $80 \%$ and $91 \%$ vs. $60 \%$ ). This metaanalysis indicates that intramuscular introduction of autologous stem cells is a feasible, safe and effective therapeutic strategy for patients with severe peripheral arterial disorders (PAD) [15]. Currently 22 phase I-III clinical trials, using ABMSCT in advanced ASO and TAO are going on. The results of these trials will provide with important information for the final consideration of stem cell therapy in these diseases [15].

Very little information is available on the long term effects of SCT that requires the clinical follow up of the patients for years [12]. Furthermore, there are no clinical data on the effects of repeated SCT neither in TAO nor in ASO. Here we report on two patients with severe Buerger's disease who received repeated ABMSCT. In both cases, the relapse occurred 30 - 36 months after the first SCT. After six months of the repeated treatment however, the clinical state of the treated limbs improved considerably, the non-healing ulcers healed more rapidly than during the first SCT without any severe adverse events. Based on these observations we conclude that repeated ABMSCT is safe and effective even in settings of repeated treatments.

Stem cell therapy carried out on the left lower limb of our first patient demonstrates an excellent clinical response to the therapy with duration of almost 4 years, despite its worse condition as compared to the right leg. Although SCT was performed with the same technique on both legs, the right limb relapsed 30 months after the treatment. We do not have a definite explanation for the clinically different local responses of this patient to the therapies performed at different time points, but it may depend on the actual composition of the functionally relevant stem- and progenitor cells. The quality control of the autologous bone marrow-derived stem- and progenitor cell suspensions performed as detailed in our previous publication [9] revealed that no major differences in the ratios of $\mathrm{CD}_{3}{ }^{+}$and $\mathrm{CD}_{3} 4^{+} \mathrm{CD} 133^{+}$subpopulations of the cell fractions used for the therapy could be detected.

Remarkably, the large and deep ischemic ulcers appeared in both cases at the same place as for the first time. This observation points to a transient and incomplete regeneration process of vessels. Nevertheless, our present results clearly show that relapsed cases of Buerger's disease can be treated by repeated ABMSCT with high efficacy without adverse events.

\section{CONCLUSION}

Stem cell therapy is a promising and prospective novel approach in the treatment of patients with severe Buerger's disease. In two cases the lower limb of patients with Buerger's disease previously treated by ABMSCT have been re-treated with freshly isolated autologous bone-marrow derived $\mathrm{CD} 34^{+}$cells. Both patients relapsed at 30 - 36 months after the first ABMSCT. Our present results clearly show that relapsed cases of advanced Buerger's disease can be treated by repeated ABMSCT with high efficacy without adverse events.

\section{ACKNOWLEDGEMENTS}

This work was supported by the EEA/Norway Grants HU-0046/ NA/2006-2/ÖP-9 entitled Development of novel stem cell-based therapies for the treatment of advanced peripheral arterial disease and by the TAMOP 4.2.2-08/1-2008-0015 EU supported NFÜ program entitled Establishing a stem cell and gene therapy. Research Centre at the University of Debrecen.

\section{REFERENCES}

[1] Ishida, A., Ohya, Y., Sakuda, H., Ohshiro, K., Higashiuesato, Y., Nakaema, M., Matsubara, M., Yakabi, S., Kakihara, A., Ueda, M., Miyagi, C., Yamane, N., Koja, K., Komori, K. and Takashita, S. (2005) Autologous peripheral blood mononuclear cell implantation for patients with peripheral arterial disease improves limb ischemia, Circulation Journal, 69, 1260-1265. doi:10.1253/circj.69.1260

[2] Irrera, G., Console, G., Martino, M., Puci, G., Morabito, F., Callea, I., Pontari, A., Marcuccio, D., Cuzzola, M., Dattola, A., Condemi, A., Messina G. and Iacopino, A.P. (2005) Therapeutic angiogenesis after infusion of bone marrow mononuclear cells for Buerger's disease: Case report, Bone Marrow Transplantation, 36, S92. 
[3] Miyamoto, K., Nishigami, K., Nagaya, N., Akutsu, K., Chiku, M., Kamei, M., Toshihiro, S., Shigeki, M., Masahiro, H., Ryoichi, T., Takeshi, N., Hiroshi, N. and Satoshi, T. (2006) Unblinded pilot study of autologous transplantation of bone marrow mononuclear cells in patients with thromboangiitis obliterans, Circulation, 114, 2679-2684.

doi:10.1161/CIRCULATIONAHA.106.644203

[4] Durdu, S., Akar, A.R., Arat, M., Sancak, T., Eren, N.T. and Ozyurda, U. (2006) Autologous bone-marrow mononuclear cell implantation for patients with Rutherford grade II-III thromboangiitis obliterans, Journal of Vascular Surgery, 44, 732-739.

doi:10.1016/j.jvs.2006.06.023

[5] Kim, D.I., Kim, M.J., Joh, Shin, J.H., Do, S.W., Moon, Y.S., Kim, J.Y., Lim, N.R., Kim, J.E., Eo, H.S., Kim, A.K., Cho, S.W., Yang, S.H., Park C.J. and Shim J.S. (2006) Angiogenesis facilitated by autologous whole bone marrow stem cell transplantation for Buerger's disease, Stem Cells, 24, 1194-1200. doi:10.1634/stemcells.2005-0349

[6] Kim, S.W., Han, H., Chae, G.T., Lee, S.H., Bo, J.H., Yoon, S., Lee, Y.S., Lee, K.S., Park H.K. and Kang, K.S. (2006) Successful stem cell therapy using umbilical cord blood-derived multipotent stem cells for Buerger's disease and ischemic limb disease animal model, Stem Cells, 24, 1620-1626. doi:10.1634/stemcells.2005-0365

[7] Kajiguchi, M., Kondo, T., Izawa, H., Kobayashi, M., Yamamoto, K., Shintani, S., Numaguchi, Y., Naoe, T., Takamatsu, T., Komori K., and Murohara, T. (2007) Safety and efficacy of autologous progenitor cell transplantation for therapeutic angiogenesis in patients with critical limb ischemia, Circulation Journal, 71, 196-201. doi:10.1253/circj.71.196

[8] Saito, Y., Sasaki, K., Katsuda, Y., Murohara, T., Takeshita, Y., Okazaki, T., Arima, K., Katsuki, Y., Shintani, S., Shimada, T., Akashi, H., Ikeda H., and Imaizumi, T. (2007) Effect of autologous bone-marrow cell transplantation on ischemic ulcer in patients with Buerger's disease”, Circulation Journal, 71, 1187-92. doi:10.1253/circj.71.1187

[9] Boda, Z., Udvardy, M., Razso, K., Farkas, K., Toth, J., Jambor, L., Olah, Zs, Ilonczai, P., Szarvas, M., Kappelmayer, J., Veréb Z., and Rajnavolgyi, E. (2009) Stem cell therapy: A promising and prospective approach in the treatment of patients with severe Buerger's disease, Clinical and Applied Thrombosis/Hemostasis, 15, 552-560. doi:10.1177/1076029608319882

[10] Wang, W., Liu, C., Qiao, T., Liu C., and Huang, D. (2010) Clinical efficacy of autologous bone marrow mononuclear cell transplantation in treating lower limb thromboangiitis obliterans, Zhongguo Xiu Fu Chong Jian Wai Ke Za Zhi, 24, 420-423.

[11] Motukuru, V., Suresh, K.R., Vivekanand, V., Raj, S. and Girija, K.R. (2008) Therapeutic angiogenesis in Buerger's disease (thromboangiitis obliterans) patients with critical limb ischemia by autologous transplantation of bone marrow mononuclear cells, Journal of Vascular Surgery, 48, 53S-60S. doi:10.1016/j.jvs.2008.09.005

[12] Matoba, S., Tatsumi, T., Murohara, T., Imaizumi, T., Katsuda, Y., Ito, M., Saito, Y., Uemura, S., Suzuki, H., Fukumoto, S., Yamamoto, Y., Onodera, R., Teramukai, S., Fukushima, M., Matsubara H. and TACT Follow-up Study Investigators (2008) Long-term clinical outcome after intramuscular implantation of bone marrow mononuclear cells (Therapeutic Angiogenesis by Cell Transplantation [TACT] trial) in patients with chronic limb ischemia, American Heart Journal, 156 1010-1018. doi:10.1016/j.ahj.2008.06.025

[13] Kakihana, A., Ishida, A., Miyagi, M., Ishiki, T., Okumura, K., Kamiyama, T., Ohya, Y. and Takishita, S. (2009) Improvement of cardiac function after granulocyte-colony stimulating factor-mobilized peripheral blood mononuclear cell implantation in a patient with non-ischemic dilated cardiomyopathy associated with thromboangiitis obliterans, Internal Medicine, 48, 1003-1007. doi:10.2169/internalmedicine.48.2117

[14] De Vriese, A.S., Billiet, J., Van Droogenbroeck J., Ghekiere J. and De Letter J.A. (2008) Autologous transplantation of bone marrow mononuclear cells for limb ischemia in a caucasian population with atherosclerosis obliterans, Journal Internal Medicine, 263, 395-403. doi:10.1111/j.1365-2796.2007.01899.x

[15] Fadini, G.P., Agostini, C. and Avogaro, A. (2010) Autologous stem cell therapy for peripheral arterial disease meta-analysis and systematic review of the literature, Atherosclerosis, 209, 10-17. doi:10.1016/j.atherosclerosis.2009.08.033

[16] Lawall, H., Bramlage, P. and Amann, B. (2010) Stem cell and progenitor cell therapy in peripheral artery disease, Thrombosis Haemostasis, 103, 696-709. doi:10.1160/TH09-10-0688

[17] Awad, O., Dedkovm, E.I. and Jiao, C. (2006) Differential healing activities of $\mathrm{CD} 34^{+}$and CD $14^{+}$endothelial cell progenitors, Arteriosclerosis Thrombosis and Vascular Biology, 26, 758-764. doi:10.1161/01.ATV.0000203513.29227.6f

[18] Zampetaki, A., Kirton, J.P. and Xu, Q. (2008) Vascular repair by endothelial progenitor cells, Cardiovascular Research, 78, 413-421. doi:10.1093/cvr/cvn081 